PROCEEDINGS OF THE

AMERICAN MATHEMATICAL SOCIETY

Volume 129, Number 6, Pages 1659-1663

S 0002-9939(00)05875-5

Article electronically published on December 13, 2000

\title{
LINE BUNDLES FOR WHICH A PROJECTIVIZED JET BUNDLE IS A PRODUCT
}

\author{
SANDRA DI ROCCO AND ANDREW J. SOMMESE
}

(Communicated by Ron Donagi)

\begin{abstract}
We characterize the triples $(X, L, H)$, consisting of line bundles $L$ and $H$ on a complex projective manifold $X$, such that for some positive integer $k$, the $k$-th holomorphic jet bundle of $L, J_{k}(X, L)$, is isomorphic to a direct sum $H \oplus \cdots \oplus H$.
\end{abstract}

\section{INTRODUCTION}

Let $X$ be a complex projective manifold. A large amount of information on the geometry of an embedding $i: X \hookrightarrow \mathbb{P}^{N}$ is contained in the "bundles of jets" of the line bundles $k \mathcal{L}=i^{*} \mathcal{O}_{\mathbb{P}^{N}}(k)$ for $k \geq 1$. The $k$-th jet bundle of a line bundle $L$ (sometimes called the $k$-th principal part of $L$ ) is usually denoted by $J_{k}(X, L)$, or by $J_{k}(L)$ when the space $X$ is clear from the context. The bundle $J_{k}(k \mathcal{L})$ is spanned by the $k$-jets of global sections of $k \mathcal{L}$. If $\alpha: \mathbb{P}\left(J_{1}(\mathcal{L})\right) \rightarrow \mathbb{P}^{N}$ is the map given by the 1-jets of elements of $H^{0}(X, \mathcal{L})$, then $\alpha\left(\mathbb{P}\left(J_{1}(\mathcal{L})\right)_{x}\right)=\mathbb{T}_{x}$, where $\mathbb{T}_{x}$ is the embedded tangent space at $x$. Similarly $\mathbb{P}\left(J_{k}(k \mathcal{L})\right)_{x}$ is mapped to the " $k$-th embedded tangent space" at $x$ (see GH for more details). Given this interpretation of the projectivized jet bundle, it is natural to expect that a projectivized jet bundle is a product of the base and a projective space only under very rare circumstances.

In this paper we analyze the more general setting where $L$ is a line bundle on $X$ (with no hypothesis on its positivity) and $J_{k}(L)=\bigoplus H$, with $H$ a line bundle on $X$. Some years ago the second author [So] analyzed the pairs $(X, L)$ under the stronger hypothesis that $J_{k}(L)$ is a trivial bundle. Though the results in this paper do not follow from $\mathrm{So}$, the only possible projective manifolds with a projectivized jet bundle of some line bundle being a product of the base manifold and a projective space, turn out to be the same, i.e., Abelian varieties and projective space. We completely characterize the possible triples $(X, L, H)$. In fact, on Abelian varieties we have the necessary and sufficient condition that $L \in \operatorname{Pic}_{0}(X)$, and if $L=\mathcal{O}_{\mathbb{P} n}(a)$, then only the range $a \geq k$ or $a \leq-1$ can occur.

Received by the editors June 30, 1998 and, in revised form, October 13, 1999 .

2000 Mathematics Subject Classification. Primary 14J40, 14 M99.

Key words and phrases. Jet bundle, complex projective manifold, projective space, Abelian variety.

The first author would like to thank the Max Planck Institute for its support.

The second author would like to thank the Max Planck Institute and the Alexander von Humboldt Foundation for its support. 
A significant amount of the research on this paper was done during our stay at the Max-Planck-Institut für Mathematik in Bonn, to which we would like to express our gratitude for the excellent working conditions.

\section{Some PRELIMinaries}

We follow the usual notation of algebraic geometry. Often we denote the direct sum of $m$ copies of a vector bundle $\mathcal{E}$, for some integer $m>0$, by $\bigoplus_{m} \mathcal{E}$. We freely use the additive notation for line bundles. We often use the same symbols for a vector bundle and its associated locally free sheaf of germs of holomorphic sections. We say a vector bundle is spanned if the global sections generate each fiber of the bundle. For general references we refer to $[\mathrm{BSo}]$ and $[\mathrm{KSp}]$.

By $X$ we will always denote a projective manifold over the complex numbers $\mathbb{C}$ and by $L$ a holomorphic line bundle on $X$.

Let $k$ be a nonnegative integer. The $k$-th jet bundle $J_{k}(L)$ associated to $L$ is defined as the vector bundle of rank $\left(\begin{array}{c}k+n \\ n\end{array}\right)$ associated to the sheaf

$$
p^{*} L /\left(p^{*} L \otimes \mathcal{J}_{\Delta}^{k+1}\right),
$$

where $p: X \times X \rightarrow X$ is the projection on the first factor and $\mathcal{J}_{\Delta}$ is the sheaf of ideals of the diagonal, $\Delta$, of $X \times X$. Let $j_{k}: H^{0}(X, L) \times X \rightarrow J_{k}(L)$ be the map which associates to each section $s \in H^{0}(X, L)$ its $k$-th jet. That means that locally, choosing coordinates $\left(x_{1}, \ldots, x_{n}\right)$ and a trivialization of $L$ in a neighborhood of $x$, $j_{k}(s, x)=\left(a_{1}, \ldots, a_{\left(\begin{array}{c}n+k \\ n\end{array}\right)}\right)$, where the $a_{i}$ 's are the coefficients of the terms of degree up to $k$ in the Taylor expansion of $s$ around $x$. Notice that the map $j_{k}$ is surjective if and only if $H^{0}(X, L)$ generates all the $k$-jets at all points $x \in X$. For example $j_{1}$ being surjective is equivalent to $|L|$ defining an immersion of $X$ in $\mathbb{P}^{h^{0}(L)-1}$.

We will often use the associated exact sequence

$$
0 \rightarrow T_{X}^{*(k)} \otimes L \rightarrow J_{k}(L) \rightarrow J_{k-1}(L) \rightarrow 0 \quad\left(j_{k}\right)
$$

where $T_{X}^{*(k)}$ denotes the $k$-th symmetric power of the cotangent bundle of $X$. There is an injective bundle map $\mathrm{KSp}$. p. 52]

$$
\gamma_{i, j}: J_{i+j}(L) \rightarrow J_{i}\left(J_{j}(L)\right) .
$$

Using the sequence $\left(j_{k}\right)$ it is easy to see that

$$
\operatorname{det} J_{k}(L)=\frac{1}{n+1}\left(\begin{array}{c}
n+k \\
n
\end{array}\right)\left(k K_{X}+(n+1) L\right) .
$$

Lemma 1.1. Let $X$ be a compact Kähler variety and $L$ a holomorphic line bundle on $X$. Then $c_{1}(L)=0$ in $H^{1}\left(T_{X}^{*}\right)$ if and only if the bundle sequence $\left(j_{1}\right)$ splits. If $c_{1}(L)=0$ in $H^{1}\left(T_{X}^{*}\right)$, then $J_{k}(L) \cong J_{k}\left(\mathcal{O}_{X}\right) \otimes L$.

Proof. A local computation, using Cech coverings, shows that the Atiyah class defined by the sequence $\left(j_{1}\right)$ is the cocycle $c_{1}(L) \in H^{1}\left(X, T_{X}^{*}\right)$. Thus $c_{1}(L)=0$ in $H^{1}\left(T_{X}^{*}\right)$ if and only if the bundle sequence $\left(j_{1}\right)$ splits.

If $c_{1}(L)=0$ in $H^{1}\left(T_{X}^{*}\right)$, then $L$ has constant transition functions. From this, the isomorphism $J_{k}(L) \cong J_{k}\left(\mathcal{O}_{X}\right) \otimes L$ follows.

Let $A$ and $B$ be vector bundles on $X$. We recall that if $\alpha \in H^{1}\left(A \otimes B^{*}\right)$ represents the vector bundle extension $E$, then any nonzero multiple $\lambda \alpha$ gives an isomorphic extension. If $\lambda=0$ this is of course false since we would get the trivial extension. It follows that: 
Lemma 1.2. Let $\lambda$ be a nonzero integer. Then $J_{1}(\lambda L) \cong J_{1}(L) \otimes(\lambda-1) L$.

Proof. Consider the extension

$$
0 \rightarrow T_{X}^{*} \otimes \lambda L \rightarrow J_{1}(L) \otimes(\lambda-1) L \rightarrow \lambda L \rightarrow 0 \quad\left(j_{1}\right) \otimes(\lambda-1) L
$$

represented by $c_{1}(L)+c_{1}((\lambda-1) L)=\lambda c_{1}(L)=c_{1}(\lambda L)$. Then, from what was observed above, $J_{1}(\lambda L)$, which is the vector bundle extension given by $c_{1}(\lambda L)$, is isomorphic to $J_{1}(L) \otimes(\lambda-1) L$.

\section{The BASIC EXAMPLES}

In this section we will characterize line bundles with splitting $k$-th jet bundles on Abelian varieties and on $\mathbb{P}^{n}$. These turn out to be the only possible examples.

Proposition 2.1. Let $L$ and $H$ be line bundles on an Abelian variety $X$. Then the following assertions are equivalent:

- $J_{k}(L) \cong \bigoplus H$ for some $k$;

- $H \cong L$ and $L \in \operatorname{Pic}_{0}(X)$.

In particular $J_{k}(L)$ splits in the sum of spanned line bundles only when $L=\mathcal{O}_{X}$ and $J_{k}(L)=\bigoplus \mathcal{O}_{X}$.

Proof. Let $X$ be an Abelian variety and assume that $J_{k}(L)=\bigoplus H$ for some line bundle $H$. Then the sequences $\left(j_{m}\right)$ for $m \leq k$ imply that there is a surjection of the trivial bundle $J_{k}(L) \otimes(-H)$ onto $L-H$. Thus the line bundle $L-H$ is a spanned line bundle with

$$
\left(\begin{array}{c}
n+k \\
n
\end{array}\right) c_{1}(L-H)=0
$$

which implies $L=H$. Using this we can find a direct summand $L$ of $J_{k}(L)$ whose image in $J_{1}(L)$ maps onto $L$ under the map $J_{1}(L) \rightarrow L \rightarrow 0$ of the sequence $\left(j_{1}\right)$. Thus the sequence $\left(j_{1}\right)$ splits and therefore $c_{1}(L)=0$ in $H^{1}\left(T_{X}^{*}\right)$ by Lemma 1.1.

Conversely assume that we have a line bundle $L \in \operatorname{Pic}_{0}(X)$. Then by Lemma 1.1, we have that $J_{k}(L) \cong J_{k}\left(\mathcal{O}_{X}\right) \otimes L$. Using the triviality of $T_{X}^{(j)}$ for all $j \geq 0$, it follows that $J_{k}\left(\mathcal{O}_{X}\right)$ is trivial.

Proposition 2.2. $J_{k}\left(\mathcal{O}_{\mathbb{P}^{n}}(a)\right)$ is isomorphic to a direct sum $\bigoplus_{(k+n)} \mathcal{O}_{\mathbb{P}^{n}}(q)$ for some $k, a, q \in \mathbb{Z}$ with $k>0$ if and only if $q=a-k$ and either $a \geq k$ or $a \leq-1$.

Proof. Let $L=\mathcal{O}_{\mathbb{P}^{n}}(a)$. First notice that if the $k$-th jet bundle splits as $J_{k}(L)=$ $\bigoplus_{\left(\begin{array}{l}k+n \\ n\end{array}\right)} \mathcal{O}_{\mathbb{P} n}(q)$ for some nonnegative $k$ and some integer $q$, then

$$
\operatorname{det} J_{k}(L)=\mathcal{O}_{\mathbb{P}^{n}}\left(\left(\begin{array}{c}
n+k \\
k
\end{array}\right)(a-k)\right),
$$

which implies that $q=a-k$. Then the fact that the $k$-th jet bundle of $\mathcal{O}_{\mathbb{P}^{n}}(a)$ always has sections for $a \geq 0$ rules out the cases $a=0, \ldots, k-1$.

Lemma 1.2 gives $J_{1}\left(\mathcal{O}_{\mathbb{P}^{n}}(a)\right) \cong J_{1}\left(\mathcal{O}_{\mathbb{P}^{n}}(1)\right) \otimes \mathcal{O}_{\mathbb{P}^{n}}(a-1)$. Then $J_{1}\left(\mathcal{O}_{\mathbb{P}^{n}}(1)\right)=$ $\bigoplus \mathcal{O}_{\mathbb{P}}$ (see, e.g., [So] ) implies

$$
J_{1}\left(\mathcal{O}_{\mathbb{P}^{n}}(a)\right)=\bigoplus_{n+1} \mathcal{O}_{\mathbb{P}^{n}}(a-1) .
$$


Dualizing $\gamma_{1,1}$ and using

$$
J_{1}\left(J_{1}\left(\mathcal{O}_{\mathbb{P}^{n}}(a)\right)\right)=J_{1}\left(\bigoplus_{n+1} \mathcal{O}_{\mathbb{P}^{n}}(a-1)\right)=\bigoplus_{n+1}\left(\bigoplus_{n+1} \mathcal{O}_{\mathbb{P}^{n}}(a-2)\right)
$$

we obtain the quotient

$$
\bigoplus_{n+1}\left(\bigoplus_{n+1} \mathcal{O}_{\mathbb{P}^{n}}(a-2)\right)^{*} \rightarrow J_{2}\left(\mathcal{O}_{\mathbb{P}^{n}}(a)\right)^{*} \rightarrow 0
$$

and thus, tensoring by $\mathcal{O}_{\mathbb{P}}(a-2)$

$$
\bigoplus_{(n+1)^{2}} \mathcal{O}_{\mathbb{P}^{n}} \rightarrow J_{2}\left(\mathcal{O}_{\mathbb{P}^{n}}(a)\right)^{*} \otimes \mathcal{O}_{\mathbb{P}^{n}}(a-2) \rightarrow 0
$$

Then the vector bundle $J_{2}\left(\mathcal{O}_{\mathbb{P}^{n}}(a)\right)^{*} \otimes \mathcal{O}_{\mathbb{P}^{n}}(a-2)$ is spanned with

$$
\operatorname{det}\left(J_{2}\left(\mathcal{O}_{\mathbb{P}^{n}}(a)\right)^{*} \otimes \mathcal{O}_{\mathbb{P}^{n}}(a-2)\right)=\mathcal{O}_{\mathbb{P}^{n}}
$$

which implies that $J_{2}\left(\mathcal{O}_{\mathbb{P}^{n}}(a)\right)^{*} \otimes \mathcal{O}_{\mathbb{P}^{n}}(a-2)=\bigoplus \mathcal{O}_{\mathbb{P}^{n}}$. Iterating this argument one gets $J_{k}\left(\mathcal{O}_{\mathbb{P}^{n}}(a)\right)^{*} \otimes \mathcal{O}_{\mathbb{P}^{n}}(a-k)=\bigoplus_{\left(\begin{array}{c}k+n \\ n\end{array}\right)} \mathcal{O}_{\mathbb{P}^{n}}$.

\section{The MAin RESUlt}

In this section we characterize complex projective manifolds having a line bundle whose projectivized $k$-jet bundle is a product of the base manifold and a projective space.

Theorem 3.1. Let $L$ and $H$ be holomorphic line bundles on $X$. Then $J_{k}(L)=$ $H \oplus \cdots \oplus H$ if and only if the triple $(X, L, H)$ is one of the two below:

(1) $(X, L, L)$ where $X$ is Abelian and $L \in \operatorname{Pic}_{0}(X)$,

(2) $\left(\mathbb{P}^{n}, \mathcal{O}_{\mathbb{P}^{n}}(a), \mathcal{O}_{\mathbb{P}^{n}}(a-k)\right)$ and $a \geq k$ or $a \leq-1$.

Proof. Assume $J_{k}(L)=H \oplus \cdots \oplus H$. Tensoring the sequence $\left(j_{k}\right)$ by $H^{*}$ gives the quotient $\bigoplus_{\left(\begin{array}{c}n+k \\ n\end{array}\right)} \mathcal{O}_{X} \rightarrow L \otimes H^{*} \rightarrow 0$. Tensoring the sequence $\left(j_{k}\right)$ by $H^{*}$ and then dualizing it gives the quotient $\bigoplus_{\left(\begin{array}{c}n+k \\ n\end{array}\right)} \mathcal{O}_{X} \rightarrow T_{X}^{(k)} \otimes\left(H \otimes L^{*}\right) \rightarrow 0$. It follows that $L \otimes H^{*}, T_{X}^{(k)} \otimes\left(H \otimes L^{*}\right)$ and thus $T_{X}^{(k)}$ are spanned bundles over $X$.

First assume that the canonical bundle $K_{X}$ is nef. Since $T_{X}^{(k)}$ is spanned, we conclude that $\operatorname{det} T_{X}^{(k)}$, which is a spanned negative multiple of $K_{X}$ is trivial. Thus $T_{X}^{(k)}$ is a trivial bundle. From this we conclude that $L-H$ is trivial. It follows that the trivial bundle $J_{k}(L)^{*} \otimes L$ has a filtration with quotient bundles $T_{X}^{(j)}$ for $0 \leq j \leq k$. This shows that $T_{X}^{(j)}$ is trivial for all $j>0$. Thus under the assumption that $K_{X}$ is nef, we conclude that $X$ would be an Abelian variety. Proposition 2.1 implies also that $L \in \mathrm{Pic}_{0}(X)$. This gives the first case of the theorem.

We are thus left with the case when $K_{X}$ is not nef. Since $T_{X}^{(k)}$ is spanned $-K_{X}$ is nef. The cone theorem then yields the existence of an extremal ray $\mathbb{R}_{+}[\gamma]$, with $1 \leq-K_{X} \cdot \gamma \leq n+1$. Let $l$ be the normalization of $\gamma$ and let

$$
T_{X \mid l}=\bigoplus \mathcal{O}_{l}\left(a_{i}\right), \quad\left(L \otimes H^{*}\right)_{l}=\mathcal{O}_{l}(b),
$$


where by abuse of notation we denote the pullback of a bundle $\mathcal{E}$ on $\gamma$ to $l$ by $\mathcal{E}_{l}$. Writing for simplicity $T_{X \mid l}^{(k)}=\left(\bigoplus_{i} \mathcal{O}_{l}\left(k a_{i}\right)\right) \oplus P$ we get

$$
T_{X \mid l}^{(k)} \otimes\left(L \otimes H^{*}\right)_{l}^{*}=\left(\bigoplus_{i} \mathcal{O}\left(k a_{i}-b\right)\right) \oplus\left(P \otimes \mathcal{O}_{l}(-b)\right) .
$$

Since $T_{X \mid l}^{(k)} \otimes\left(L \otimes H^{*}\right)_{l}^{*}$ is spanned we conclude that $k a_{i} \geq b \geq 0$. Note that $b>0$. Indeed if $b=0$, then

$$
0=\operatorname{deg}\left(\operatorname{det} J_{k}(L)_{l} \otimes\left(-H_{l}\right)\right)=\frac{1}{n+1}\left(\begin{array}{c}
n+k \\
n
\end{array}\right) k K_{X} \cdot l \neq 0 .
$$

Thus $a_{i}>0$ for all $i$ 's. Moreover from sheaf injection $0 \rightarrow T_{l} \rightarrow T_{X \mid l}$ we see that $T_{X \mid l}$ must contain a factor $\mathcal{O}_{l}\left(a_{i}\right)$ with $a_{i} \geq 2$. Then $-K_{X} \cdot \gamma=\sum_{i=1}^{n} a_{i} \leq n+1$ implies that $a_{j}=2$ for one $j$ and $a_{i}=1$ for $i \neq j$, i.e., $-K_{X} \cdot \gamma=n+1$. Now from Mori's proof of Hartshorne conjecture (see $[\mathrm{L}, \S 4]$ ) we deduce that $X$ must be $\mathbb{P}^{n}$. At this point we have recovered case (2) by applying Proposition 2.2 .

Propositions 2.1 and 2.2 show that if we are in cases (1) and (2) respectively, then $J_{k}(L)=H \oplus \cdots \oplus H$ is satisfied.

\section{REFERENCES}

[BSo] M.C. Beltrametti and A.J. Sommese, The Adjunction Theory of Complex Projective Varieties, Expositions in Math. 16 (1995), W. de Gruyter, Berlin. MR 96f:14004

[GH] P. Griffiths and J. Harris, Algebraic geometry and local differential geometry, Ann. Sci. Ècole Norm. Sup. (4) 12 (1979), 355-432. MR 81k:53004

[KSp] A. Kumpera and D. Spencer, Lie Equations; Vol. I: General Theory, Ann. of Math. Stud. 73 (1972), Princeton University Press, Princeton, N.J. MR 52:1805

[L] R. Lazarsfeld, Some applications of the theory of positive vector bundles, Complete Intersections, CIME course 1983, Acireale (Catania) ed. by S. Greco and R. Strano, Lecture Notes in Math. 1092 (1984), 29-61, Springer-Verlag, New York. MR 86d:14013

[So] A.J. Sommese, Compact Complex Manifolds possessing a line bundle with a trivial jet bundle, Abh. Math. Sem. Univ. Hamburg 47 (1978), 79-91. MR 58:17231

Department of Mathematics, KTH, Royal Institute of Technology, S-100 44 StockHOLM, SWEDEN

E-mail address: sandra@math.kth.se

URL: http://www.math.kth.se/ sandra

Current address: Department of Mathematics, Yale University, P.O. Box 208229, New Haven, Connecticut 06520

Department of Mathematics, University of Notre Dame, Notre Dame, Indiana 46556

E-mail address: sommese@nd.edu

$U R L:$ http://www.nd.edu/ sommese 